\title{
Should Pulsatile Preservation Be the Gold Standard in Kidney Transplantation?
}

\author{
Maria Letizia Lo Faro ${ }^{1} \cdot$ M. Zeeshan Akhtar ${ }^{1} \cdot$ Catherine Boffa $^{1} \cdot$ Rutger Ploeg $^{1}$
}

Published online: 15 April 2015

(C) Springer International Publishing AG 2015

\begin{abstract}
In recent years, dramatic improvements in kidney transplantation, together with a rising incidence of diseases such as diabetes, have led to an increasing demand for deceased donor kidneys for transplantation. Hence, it has been necessary to expand the kidney donor pool by using organs once considered unsuitable for transplantation. These higher risk kidneys are typically from older donors with additional comorbidities and are more susceptible to injury. Therefore, the transplant community has been focusing efforts in trying to improve the outcomes of these high-risk organs. Preservation by pulsatile machine perfusion has been associated with decreased risk of delayed graft function and renoprotective effects on deceased donor kidneys. The aim of this review is to provide an overview of the principles of this preservation technique and to review the evidence regarding its usage for deceased donor kidneys compared to standard static cold storage.
\end{abstract}

Keywords Hypothermic machine perfusion - Pulsatile flow . Deceased donor kidney transplantation $\cdot$ Extended criteria donors $\cdot$ Delayed graft function $\cdot$ Static cold storage

This article is part of the Topical Collection on Kidney Transplantation

Rutger Ploeg

rutger.ploeg@nds.ox.ac.uk

Maria Letizia Lo Faro

letizia.lofaro@nds.ox.ac.uk

M. Zeeshan Akhtar

zeeshan.akhtar@nds.ox.ac.uk

Catherine Boffa

catherine.boffa@nds.ox.ac.uk

1 Oxford Transplant Centre, Nuffield Department of Surgical Sciences, Churchill Hospital, University of Oxford, Oxford OX3 7LE, UK

\section{Introduction}

Over the past 10 years, there has been a great improvement in kidney transplantation, which has been paralleled by an increasing demand for donor organs, due to the higher incidence of diseases which can cause kidney failure, such as hypertension and diabetes [1]. Thus, there has been the development of a significant disparity between organ supply and clinical need. With a limited supply, many transplant centers are now accepting kidneys from older donors or donors with additional medical comorbidities, such as hypertension (these donors are generally referred to as extended criteria donors, ECDs) and from donors declared dead following circulatory arrest (DCDs) [1]. These organs have an increased sensitivity to cold and warm ischemic injury and may benefit from optimized resuscitative ex situ organ preservation techniques [2], which limit the damage these organs are subjected to upon storage and reperfusion injury.

Most organs are currently preserved by static cold storage (CS). However, many studies have demonstrated advantages of (pulsatile) machine perfusion (MP) over CS preservation for kidney transplantation $[2-5,6 \bullet \bullet, 7 \bullet]$, especially in the case of higher risk organ donors, such as ECDs and DCDs.

The first attempts at perfusing isolated organs date as far back as the late nineteenth century and beginning of the twentieth century, when isolated organs were perfused with small pumps using whole blood [2]. In the 1960s, before the introduction of criteria to define neurological death (brain death, $\mathrm{BD}$ ), all deceased donor kidneys were procured following circulatory arrest, and pulsatile preservation was used then in order to limit the injury caused by cold ischemia [1]. This approach was refined by the work of F. Belzer and others, who combined the use of continuous perfusion and hypothermic storage and substituted whole blood with microfiltered cryoprecipitated plasma (CPP) [2]. In 1967, Belzer et al. 
showed that canine kidneys could be successfully preserved for 24 and $72 \mathrm{~h}$ using oxygenated CPP and pulsatile MP [8].

Further progress in pulsatile perfusion research was slowed down by the introduction of donation after brain death (DBD) criteria and the development of a novel preservation solution (Collins' solution). Introduction of DBD criteria allowed for organs to be retrieved prior to circulatory arrest, minimizing the warm ischemic time. At the same time, the development of Collins' solution allowed for the successful transplantation of kidneys after simple static $\mathrm{CS}$, during which the organs were kept submerged in the solution at $0-4{ }^{\circ} \mathrm{C}$ in a box with melting ice. Evidently at the time, this type of simple preservation represented an advantage, compared to the big (and expensive) perfusion machines (Fig. 1). Concurrently, advances in immunosuppressive therapies were made and transplant outcomes improved significantly, independently from the preservation technique used. At the time, in an era with young and relatively healthy donors without major comorbidities, studies investigating transplantation outcomes failed to find any advantage derived from pulsatile MP compared to CS. In addition, the costs and availability of pumping devices at the time made kidney preservation by CS the standard method for ex situ preservation of kidneys and most other organs (Fig. 1).

This situation has changed. To date, with a shift in age and comorbidity of organ donors, more ECDs and higher risk organs are being used for transplantation, with almost $50 \%$ of organs retrieved from DCDs [1]. Attention is once again focusing on the quality of organ preservation
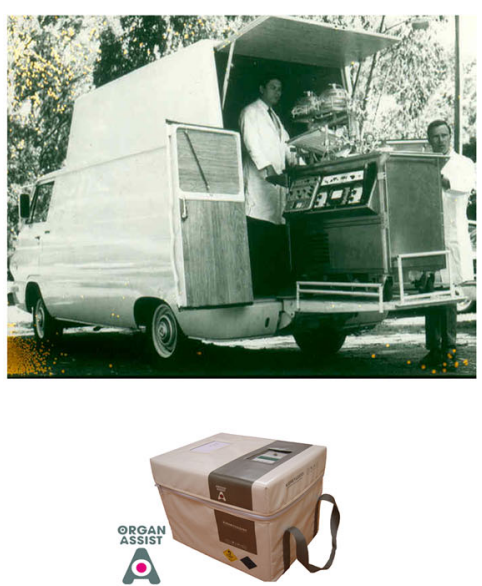

Hypothermic machine perfusion with oxygen and a search to develop novel organ preservation techniques using MP.

\section{Principles and Mechanisms of Machine Perfusion}

Many preservation techniques including static CS and MP are based on preservation of the organs in hypothermia by suppression of the organ metabolism. It is estimated that cooling an organ to $4{ }^{\circ} \mathrm{C}$ will reduce the metabolic rate to $10 \%$ of that at normal body temperature. Hypothermia and reduced supply of $\mathrm{O}_{2}$ to the organs cause a metabolic switch from aerobic to anaerobic pathways, in order to still maintain energy production and tissue function. Anaerobic pathways (e.g., glycolysis) induce tissue acidosis, alongside reduced production of ATP (if compared to aerobic pathways). In the long term, these effects are deleterious. Reduced ATP levels compromise the functioning of membrane ion channels, which causes cell swelling and edema. Reduced $\mathrm{O}_{2}$ and impaired cellular respiration will also cause electron leakage from the mitochondria and production of dangerous free radicals and reactive oxygen species. To counteract these effects, the organ preservation solutions are often supplemented with large colloid inert molecules, to maintain organ osmotic pressure, buffers to prevent acidosis; anti-oxidants to limit free radical-mediated damage, and substrates to support ATP regeneration.

In the case of static CS, during organ procurement, flushing of the kidneys removes blood and replaces it with a buffered preservation solution (e.g., University of Wisconsin solution, UW), generally containing anti-oxidants, colloids, and large
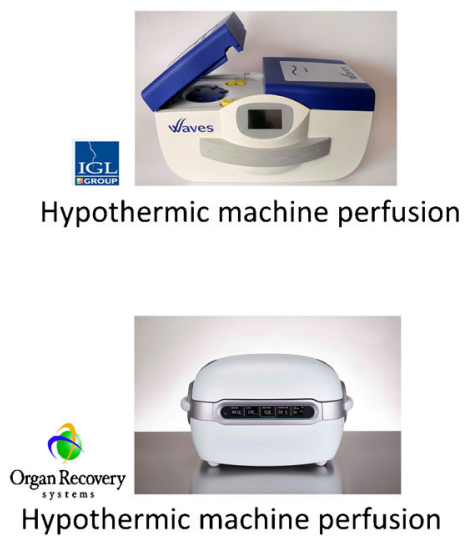

Fig. 1 Comparison between the early prototype and current machines used for hypothermic pulsatile kidney preservation. Above left: The original "transportable" Belzer machine as one of the earlier prototypes of pumps for kidney machine perfusion, allowing for the first time transportation of kidneys from the distant donor hospital to the actual transplant center. Above right and below: Examples of "portable" machine perfusion pumps currently approved for clinical use (Waters
Medical Systems (IGL), Organ Assist, Organ Recovery Systems). The development of these easily transportable and miniaturized devices contributed to renew the interest in hypothermic machine perfusion of kidney for transplantation. Images of the perfusion machines are reproduced with permission from IGL, Organ Assist, and Organ Recovery Systems 
inert molecules, as described before [2,9]. The organs are then statically stored in cold preservation solution (Fig. 2).

MP preserves organs in a more physiological manner. Similarly to CS, hypothermic machine perfusion (HMP) slows down the organ metabolism, thus reducing nutrient and $\mathrm{O}_{2}$ requirements. However, the perfusion also allows for the continuous supply of nutrients to the organs (which can be added to the perfusate) in order to support energy production, both in the presence or absence of $\mathrm{O}_{2}$ (oxygenated or non-oxygenated $\mathrm{MP}$ ), while other toxins and free radicals produced during cold or warm ischemia (in the case of DCDs) can be flushed out (Fig. 2). The circulation of the perfusate is produced by pumps that can generate either a continuous or a pulsatile flow. HMP also has the additional benefits of being able to decrease vasospasm and provide additional information, such as flow rate and renal vascular resistance that can be used to better evaluate organ viability and functionality prior to transplantation $[2,10]$.

MP preservation, by providing nutrients and eliminating toxic metabolites with the perfusate flow, renders kidneys more resilient to the effects of ischemia/reperfusion injury (Fig. 3). The actual cellular mechanisms behind how this protection occurs are currently poorly understood. Of interest, pulsatile flow, alongside reproducing a more physiological setting, has also been associated with the expression of flowdependent vasoprotective endothelial genes $[2,11]$ and reduced expression of inflammatory genes [12]. In particular, Kruppel-like factor 2 (KLF2) seems to play a critical role through the inhibition of pro-inflammatory responses, the production of vasodilators, specifically endothelial nitric oxide,

\section{Flush out blood \\ Cold storage solution}

Cool to $0-4^{\circ} \mathrm{C}$

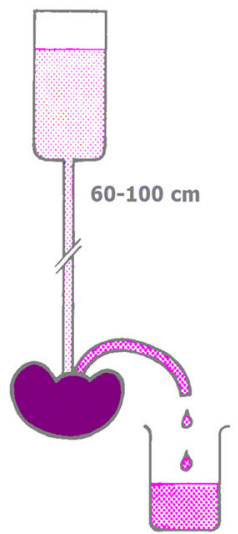

Fig. 2 Schematic representation of static cold storage and machine perfusion. Left: before static cold storage, blood is flushed out of the kidneys with cold preservation solution. The organ is then submerged in preservation fluid and preserved in a box on melting ice $\left(0-5^{\circ} \mathrm{C}\right)$. Right: prior to machine perfusion, the kidney is flushed out and then connected with its artery to the perfusion circuit and submerged in a cassette filled with preservation solution $\left(5^{\circ} \mathrm{C}\right)$. The solution is pumped and the expression of anti-thrombogenic mediators (e.g., thrombomodulin) [11]. The shear-dependent stimulation of the vasculature in the preserved organ can also be obtained by continuous flow, but studies have shown improved circulation and organ function when pulsatile perfusion was used (with evidence collected for both liver and kidney) [13, 14•]. In the latter study, Gallinat et al. used an isolated kidney perfusion model in pigs with either pulsatile or non-pulsatile flow and compared it with CS-preserved grafts. Compared with CS, pulsatile MP but not continuous flow MP significantly improved markers of renal function, such as renal perfusate flow and urine production. It also significantly enhanced the reduction of creatinine and urea levels in the perfusate, following reperfusion. Perfusate levels of fatty acid binding protein, a marker of tubular cell injury, were also dramatically reduced by pulsatile perfusion, not by continuous flow perfusion [14•]. The pulsatile flow induced a significant mRNA elevation of KLF2 and endothelial nitric oxide synthase, along with significantly higher perfusate levels of nitric oxide.

As mentioned before, machine perfusion can be performed in the presence or absence of $\mathrm{O}_{2}$. Oxygenation can be achieved by direct persufflation of the organ, by dissolving $\mathrm{O}_{2}$ in the perfusate or by using $\mathrm{O}_{2}$ carriers in solution. It has been shown that by oxygenated hypothermic perfusion, it is possible to restore kidney ATP levels $[15,16]$, which will be able to support normal cellular function, prevent cell swelling, and cell death. Interestingly, it has been found that if kidneys were subjected to warm ischemia, it was only possible to recover their ATP levels by oxygenated perfusion and not by $\mathrm{CS}$, while there was no significant difference in the case of
Machine Perfusion 5-10 ${ }^{\circ} \mathrm{C}$

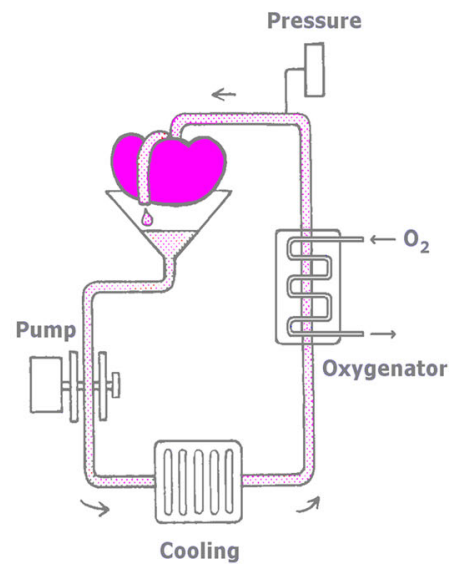

through the renal artery, while the effluent from the vein is collected in a well and recirculated. Depending on the respective machine, it will regulate flow speed, pressure, and temperature, which can also be monitored in real time. Pumps can either produce a continuous or pulsatile flow, and in some devices, the perfusion solution can be persufflated with $\mathrm{O}_{2}$ (oxygenator) during hypothermic oxygenated MP 
Fig. 3 Potential protective mechanisms during pulsatile machine perfusion. The protective mechanisms of machine perfusion are currently poorly understood, but some pathways have emerged as being potentially beneficial. By supplementing the perfusate with $\mathrm{O}_{2}$ and nutrients, it is possible to support the kidney basal metabolic requirements and ATP levels. In parallel, toxins and free radicals are eliminated with the perfusate and do not accumulate in the organ. Vasodilatory and protective endothelial genes are expressed during pulsatile MP and play a role in protecting the vasculature and decreasing vasospasm

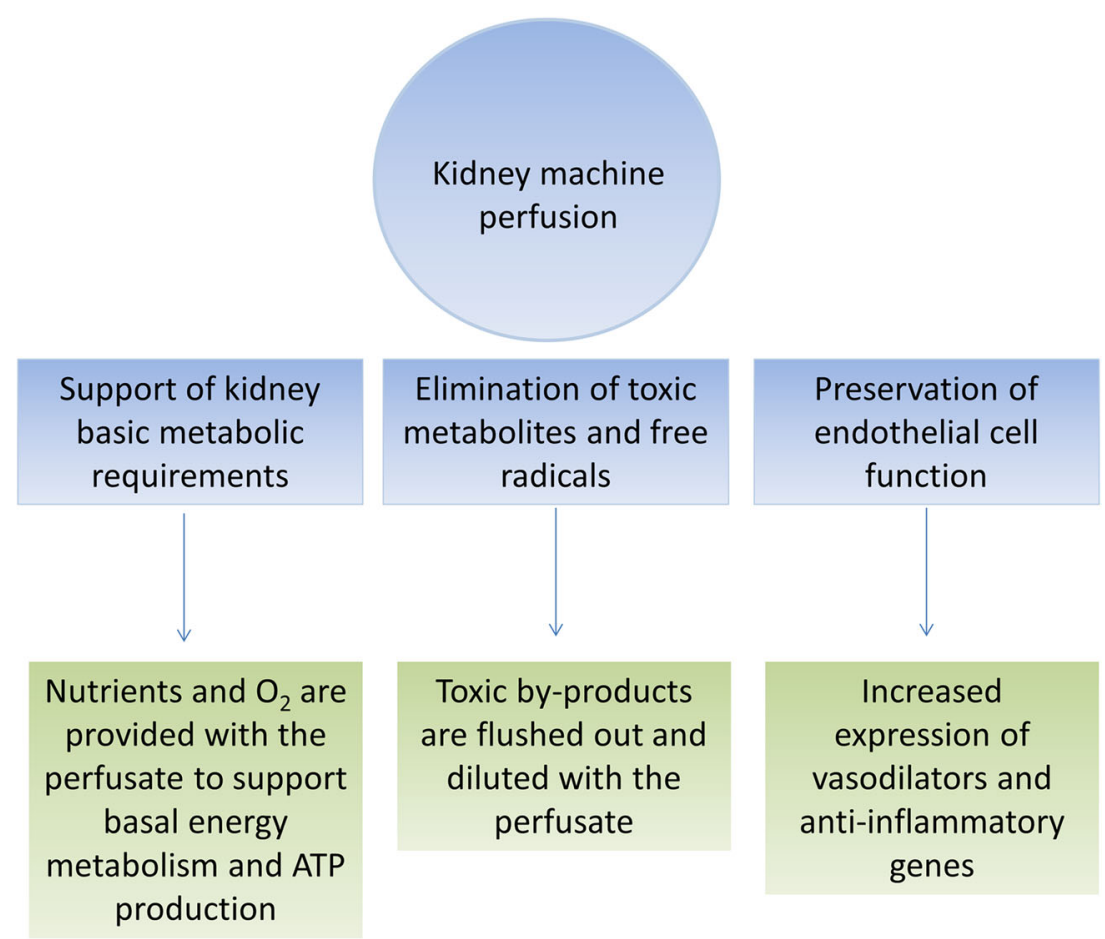

kidneys not subjected to warm ischemia [15]. Although in hypothermia organ metabolism is slowed down, it is known that the organs would still consume $\mathrm{O}_{2}$, and it is possible to speculate that oxygenated HMP would be beneficial for the preservation of ischemic organs by supporting cellular metabolism and regeneration of ATP.

Therefore, either by modulation of anti-inflammatory and vasodilatory responses or by restoration of tissue ATP levels, these studies provide additional findings in support of the beneficial effects of MP, especially in the case of higher risk organs (Fig. 3).

Experimental models are also currently investigating the effects of temperature on MP of kidneys. Normothermic and subnormothermic machine perfusions have been found to improve kidney function, in animal models [29, 30]. Although normothermic and subnormothermic temperatures can prevent the deleterious effects of cooling the organs and are, in a way, more physiological, they are also associated with higher metabolic demands, so appropriate preservation solutions need to be designed, in order to provide the organs with sufficient $\mathrm{O}_{2}$ and nutrients.

\section{Clinical and Pre-clinical Studies}

As previously mentioned, the initial clinical studies did not report significant differences in graft survival when comparing MP and CS. However, many of these were small, retrospective, non-randomized studies and often not powered enough to see any significant differences in such heterogeneous populations of kidney donors and recipients. Even 10 years ago, Wight et al. published a systematic review in which they analyzed the effectiveness of MP and CS in reducing delayed graft function (DGF) and potentially improving graft survival in recipients of kidneys from both DBDs and DCDs [17]. The studies selected covered a 30year period (from 1971 to 2002) and were mostly small, but the evidence presented suggested that MP was able to lead to a $20 \%$ reduction in the incidence of DGF, both in DBDs and DCDs. No statistical significant difference in graft survival was found, based on aggregate results of randomized controlled trials [17].

Similarly, other studies (both clinical and pre-clinical) later reported that MP was associated with lower DGF and also with lower discard rates of ECD organs. Schold et al. [3] conducted a retrospective study in which they analyzed the patterns of MP use by interrogating the US Scientific Registry of Transplant Recipients (SRTR) database for all deceased donor kidney transplants which took place from 1994 to 2003. The authors found that the organ utilization rates for standard criteria donors were similar by storage type (CS vs. $\mathrm{MP})$, but for ECD organs, there was a significantly higher $(p<0.001$, MP vs CS) utilization rate when MP was used to preserve these organs [3]. The use of MP appeared to be quite variable across all transplant centers, but throughout DGF rates were significantly lower with MP preservation, compared to CS. Additionally, MP was associated with a benefit on death-censored graft survival [3]. These findings and more specifically the beneficial effects of MP on ECD organs were also confirmed by other studies (retrospective or small 
prospective controlled studies) that once again reported reduced incidence of DGF, reduced return to dialysis post-transplantation, and enhanced utilization of ECD kidneys, when MP was used [4, 18-21].

Similarly, potential post-operative beneficial effects of MP preservation were also found for DCD donors [22, 23, 25] and in experimental animal models of DCD [24]. As found in ECD donors, it was possible to observe improvements in early transplantation outcomes, such as DGF, when comparing MP preservation with CS of kidneys from DCD donors [22, 23, 25], despite the fact that this type of donor is generally more at risk of developing DGF and other post-transplant complications [25]. Authors additionally found shorter hospitalization times and improved post-operative ( 7 and 30 days) and early (6 and 12 months) graft function for MP-preserved kidneys compared to CS $(p=0.05)$ [23]. Improved renal function upon MP preservation was also found for an experimental pig model of warm ischemia damaged organ, in which the warm ischemia damaged grafts preserved with MP showed kidney functions comparable to that of the non-ischemic controls [24]. Despite the findings from the studies cited above, a UK multicenter randomized controlled trial [26] showed that MP offered no advantages over CS (in terms of DGF incidence and graft and patient survival), for kidneys from controlled DCD donors, with mean warm and cold ischemic times of around $15 \mathrm{~min}$ and $14 \mathrm{~h}$, respectively. The differences in the studies might be due to the different warm ischemic times that occurred before organ procurement, as MP might be of greater benefits in the case of longer warm ischemia. Also, in the study by Watson et al. [26] MP was not started straight after retrieval (as it was in other studies mentioned), but the organs were first preserved by static CS, then followed by MP (delayed MP) prior to transplant. The use of immediate MP throughout the cold ischemic period may be necessary to achieve a benefit [26].

Limitations of the above-cited works are that most of them are retrospective studies that rely on a relatively limited number of patients and are only based on the information made available from the centers (i.e., there is a potential risk of inaccurately recorded ischemic times). These types of retrospective studies cannot fully address whether the beneficial effects observed are intrinsic to MP or have more to do with its logistics and diagnostics value, such as the discard of poorly performing kidneys based on their pump parameters, which help in the evaluation of organ viability before transplantation (as previously described).

The first multicenter, prospective, randomized, highvolume clinical study comparing MP and CS preservation was published by Moers et al. in 2009 [5]. In this international randomized controlled trial, one kidney from 336 consecutive deceased donors was assigned to MP and the contralateral kidney to CS. All 672 recipients were then followed up for 1 year. The primary end point of the study was DGF (defined as the need of dialysis in the first week after transplantation). Secondary end points were the duration of DGF, DGF defined by the rate of decrease in serum creatinine level, primary nonfunction, serum creatinine level and clearance, acute rejection, length of hospitalization, and finally allograft and patient survival [5]. The study showed that MP significantly reduced the risk of DGF. Indeed, DGF developed in 70 patients in the MP group, against 89 in the CS group $(p=0.01)$. MP was also shown to significantly enhance the rate of the decrease in serum creatinine levels, reduce the duration of DGF, and was associated with lower serum creatinine levels during the first 2 weeks post-transplantation [5]. MP was also associated with a reduced risk of graft failure $(p=0.03)$ and superior 1year allograft survival $(p=0.04)[5]$. The study did not select for a specific type of donor; therefore, it was able to investigate a scenario of "everyday practice" for a transplant center. In the same context, a separate and sufficiently powered study was performed by the same group to evaluate the impact of MP vs. CS in ECDs [27]. In the study, the authors included 91 consecutive deceased ECDs, and once again, one kidney was randomized to MP and the contralateral to CS. Using a logistic regression model, MP was shown to significantly reduce the risk of DGF compared with CS ( $p=0.047)$. Additionally, the incidence of primary non-function in the CS group was higher than in the MP group $(p=0.04)$, while 1-year graft survival was significantly higher in MP kidneys compared to coldstored kidneys ( $p=0.02)$. As many retrospective studies have previously found beneficial effects of MP on short-term outcomes, but not on overall long-term graft function and survival, the authors also decided to extend the recipient follow-up period to 3 years [6]. New end points were 3-year graft survival, patient survival, and serum creatinine level. Overall, the 3-year graft survival was better for MP kidneys, compared to CS (91 vs. $87 \%$ CS, $p=0.04$ ) [6••]. Interestingly, the 3-year graft survival after MP was better than that after CS for DBD, but not for DCD kidneys and was most pronounced in the case of ECDs. DGF was found to be associated with a lower rate of graft survival of kidneys donated after brain death $[6 \bullet \bullet$, so, as MP has been shown to reduce the risk of DGF, it is not surprising to consequently observe increased graft survival of these organs. The fact that the same protective effects are not seen for DCD kidneys might relate to the presence of different mechanisms of injury contributing to a different type of DGF in these donor organs, compared to DBDs and ECDs. Also, the beneficial effects of MP might be more apparent in the case of ECDs, due to their older age and reduced regenerative capacities. As we are now moving towards a scenario with more ECD donors, but also older DCDs, it is possible to speculate that perhaps the protective effects of MP might become more evident now, with an aging donor population.

The above studies and other prospective studies were recently included in a systematic review and meta-analysis comparing MP and CS effects on kidney transplant outcomes 
(both short and long term) [28]. Eighteen studies were included: seven randomized clinical trials (1475 kidneys) and 11 non-randomized clinical trials (728 kidneys), which were analyzed separately. The non-randomized clinical trials all had different definitions of DGF (and some did not define it at all), so discussion will focus on the randomized trials. The randomized trials showed a statistically significant reduction in DGF following MP $(p=0.002)$ [28]. Additionally, when stratified by donor type (DBD, DCD, and ECD), MP appeared to be likely to reduce DGF for all donors, but statistical significance was not reached (perhaps due to lack of statistical power because of the small number of donors for each group). There was no effect of MP on short-term or longer term renal function, but this might have been confounded by the fact that already a large number of patients in the CS group developed DGF in these studies. Other secondary outcomes (such as primary non-function, acute rejection, and patient survival) were not affected in this study [28], but the number of studies included in the work was relatively small, and so it is unlikely to have adequate statistical power to identify differences in secondary outcomes.

Recently, Gill et al. have conducted a Scientific Registry of Transplant Recipients database-based study, examining the association of MP with DGF in a very large cohort of patients $(n=94,709)$ from the USA, who underwent deceased donor kidney transplants between 2000 and 2011 [7•]. Within each donor group examined (DBD, ECD, and DCD), transplants were stratified based on duration of cold ischemia time (CIT) grouped in cohorts of $6 \mathrm{~h}$ (from 0 to $6 \mathrm{~h}, 6$ to $12 \mathrm{~h}$ to $>36 \mathrm{~h}$ ). The odds of DGF were lower with MP across all CIT in DBD transplants, when CIT was $>6 \mathrm{~h}$ in ECDs, and when CIT was between 6 and $24 \mathrm{~h}$ in DCDs [7•]. The duration of cold ischemia was found to be independently associated with a higher risk of DGF, irrespective of the preservation method, and although this was significantly affected by MP (reduction in the risk of DGF), the association was not eliminated completely [7•]. The use of a large cohort of patients is definitely a positive aspect of this study, but there are limitations, such as the fact that no duration of DGF is reported and that the authors did not investigate any other secondary end points in this work.

\section{Conclusion}

The existing gap between organ supply and demand has led to a more extensive use of marginal organ donors for transplantation, such as ECDs and DCDs. These organs are often from older donors and are generally more susceptible to injury. For example, it has been shown that they have a higher risk of developing DGF post-transplantation, which is then correlated to higher rates of allograft dysfunction, acute rejection, and lower patient survival $[2,7 \bullet]$. The increasing use of these marginal donor organs has contributed to boost the interest of the transplant community to research and develop novel organ preservation techniques, which can maintain organ viability and functionality more efficiently than static CS. Hypothermic machine perfusion (HMP) involves cannulation of the renal artery after flush-out of the retrieved organ, so that cold preservation fluid (oxygenated or not) can be supplied in a pulsatile manner (or continuously) in a pump-supported system. As discussed above, several prospective and retrospective studies have shown that the use of HMP correlates to lower risks of DGF compared to CS $[4,5,6 \bullet \bullet, 7 \bullet]$. From a molecular point of view, the beneficial effects of MP seem to be correlated with increased expression of anti-inflammatory and vascular protective genes, increased production of nitric oxide to support vasodilation and microcirculation, and decreased expression of inflammatory mediators $[11-13,14 \bullet]$. Additionally, oxygenation and the continuous provision of nutrients are able to support the restoration of tissue ATP levels $[15,16]$ and consequently preserve organ functionality during cold ischemia.

Despite the fact that most studies show a significant decrease in DGF with MP for all the donor types, only a few have been able to show statistically significant beneficial effects on long-term graft function and survival $[5,6 \bullet \bullet, 27]$, which is why it is still debated whether MP should be the organ preservation method of choice for deceased donor kidney transplantation. There is a need for further studies to address this question, as many of the studies which failed to find a correlation between MP and improved long-term graft function did show a trend towards improvement and might have been limited by the lack of statistical power. The same goes for retrospective and meta-analysis studies, which so far have managed to include only a limited number of data/studies, which also often presented different definitions of DGF and donor selection criteria.

One of the main criticisms against the routine use of MP is that it is not cost-effective, since it requires the supervised use of specific perfusion machines. In reality, even if future clinical studies fail to prove a significant improvement in longterm graft function, it is fair to say that by reducing DGF, MP would help in reducing the length of patients' hospitalization and their need for dialysis, thereby offsetting a significant amount the cost associated with it. Indeed, this has been confirmed by a recent study by Groen et al. [31], who have performed a cost-effectiveness study of MP vs. CS, finding costsavings of around $\$ 86,000$ per life-year gained in favor of MP [31].

In conclusion, there is strong clinical and experimental evidence, presented in various studies and reviewed in this work, describing the beneficial effects of machine perfusion on organ preservation and protection from injury. MP should be considered as the gold standard for higher risk kidney preservation, but at the moment, there are probably not enough 
clinical and mechanistic studies supporting the use of a pulsatile flow. Although we might still be a step behind its routine and widespread application for kidney preservation, pulsatile MP is an attractive route to further explore, alongside regulation of the temperature and $\mathrm{O}_{2}$ tensions, in order to rescue marginal organs, better their functionality, and improve transplantation outcomes.

\section{Compliance with Ethics Guidelines}

Conflict of Interest Maria Letizia Lo Faro, M. Zeeshan Akhtar, and Catherine Boffa declare that they have no conflict of interest.

Rutger Ploeg gives occasional advice to Bridge to Life Inc. and Teva on transplant-related matters. This work is not in conflict with the requested review submitted to the journal.

Human and Animal Rights and Informed Consent All the studies involving human participants reported in this review were in accordance with the ethical standards of the institutional and/or national research committee and with the 1964 Helsinki declaration and its later amendments or comparable ethical standards.

\section{References}

Papers of particular interest, published recently, have been highlighted as:

- Of importance

•• Of major importance

1. Gritsch H. Pulsatile perfusion - time for a prospective trial. Nat Rev Nephrol. 2014;10:191-2.

2. Yuan X, Theruvath A, Ge X, et al. Machine perfusion or cold storage in organ transplantation: indication, mechanisms, and future perspectives. Transpl Int. 2010;23(6):561-70.

3. Schold J, Kaplan B, Howard R, et al. Are we frozen in time? Analysis of the utilization and efficacy of pulsatile perfusion in renal transplantation. Am J Transplant. 2005;5:1681-8.

4. Shah A, Milgrom D, Mangus R, et al. Comparison of pulsatile perfusion and cold storage for paired kidney allografts. Transplantation. 2008;86:1006-9.

5. Moers C, Smits J, Maathuis M-H, et al. Machine perfusion or cold storage in deceased-donor kidney transplantation. N Engl J Med. 2009;360:7-19.

$6 . \bullet$ Moers C, Pirenne J, Paul A, et al. Machine perfusion or cold storage in deceased-donor kidney transplantation. N Engl J Med. 2012;366: 8. First multi-center, prospective, randomized clinical study providing evidence for increased long-term graft survival with MP.

7. Gill J, Dong J, Eng M, et al. Pulsatile perfusion reduces the risk of delayed graft function in deceased donor kidney transplants, irrespective of donor type and cold ischemic time. Transplantation. 2014;97:668-74. High-volume retrospective study showing decreased DGF with MP. MP was also shown to reduce the effects of cold ischemic times.

8. Belzer F, Ashby B, Dunphy J. 24-hour and 72-hour preservation of canine kidneys. Lancet. 1967;2:536-8.
9. Ploeg R, Goossens D, Vreugdenhil P, et al. Successful 72-hour cold storage kidney preservation with UW solution. Transplant Proc. 1988;20:935-8.

10. St Peter S, Imber C, Friend P. Liver and kidney preservation by perfusion. Lancet. 2002;359:604-13.

11. Chatauret N, Coudroy R, Delpech P, et al. Mechanistic analysis of nonoxygenated hypothermic machine perfusion's protection on warm ischemic kidney uncovers greater eNOS phosphorylation and vasodilation. Am J Transplant. 2014;14:2500-14.

12. Mas V, Archer K, Dumur C, et al. Reduced expression of inflammatory genes in deceased donor kidneys undergoing pulsatile pump preservation. PLoS One. 2012;7(4):e35526.

13. Luer B, Fox M, Efferz P, et al. Adding pulsatile vascular stimulation to venous systemic oxygen persufflation of liver grafts. Artif Organs. 2014;38(5):404-10.

14. Gallinat A, Fox M, Luer B, et al. Role of pulsatility in hypothermic reconditioning of porcine kidney grafts by machine perfusion after cold storage. Transplantation. 2013;96:538-42. The study presents evidence for the beneficial role of pulsatile flow in organ preservation being modulated by decreased expression of inflammatory genes and increased expression of vasculoprotective enzymes.

15. Buchs JB, Lazeyras F, Ruttimann R, et al. Oxygenated hypothermic pulsatile perfusion versus cold static storage for kidneys from non heart-beating donors tested by in-line ATP resynthesis to establish a strategy of preservation. Perfusion. 2011;26(2):159-65.

16. Lazeyras F, Buhler L, Vallee JP, et al. Detection of ATP by "in line" ${ }^{31} \mathrm{P}$ magnetic resonance spectroscopy during oxygenated hypothermic pulsatile perfusion of pigs' kidneys. Magn Reson Mater Phy. 2012;25:391-9.

17. Wight J, Chilcott J, Holmes M, et al. Pulsatile machine perfusion vs. cold storage of kidneys for transplantation: a rapid and systematic review. Clin Transpl. 2003;17:293-307.

18. Stratta R, Moore P, Farney A, et al. Influence of pulsatile perfusion preservation on outcomes in kidney transplantation from expanded criteria donors. J Am Coll Surg. 2007;204:873-84.

19. Matsuoka L, Almeda J, Mateo R. Pulsatile perfusion of kidney allografts. Curr Opin Organ Transpl. 2009;14:365-9.

20. Abboud I, Antoine C, Gaudez F, et al. Pulsatile perfusion preservation for expanded-criteria donors kidneys: impact on delayed graft function rate. Int J Artif Organs. 2011;34(6):513-8.

21. Cannon R, Brock G, Garrison N, et al. To pump or not to pump: a comparison of machine perfusion vs cold storage for deceased donor kidney transplantation. J Am Coll Surg. 2013;216:625-34.

22. Moustafellos P, Hadjianastassiou V, Roy D, et al. The influence of pulsatile preservation in kidney transplantation from non-heartbeating donors. Transplant Proc. 2007;39:1323-5.

23. Plata-Munoz J, Muthusamy A, Quiroga I, et al. Impact of pulsatile perfusion on postoperative outcome of kidneys from controlled donors after cardiac death. Transpl Int. 2008;21(9):899-907.

24. Schreinemachers M, Doorschodt B, Florquin S, et al. Pulsatile perfusion preservation of warm ischaemia-damaged experimental kidney grafts. Br J Surg. 2010;97:349-58.

25. Lodhi S, Lamb K, Uddin I, et al. Pulsatile pump decreases risk of delayed graft function in kidneys donated after cardiac death. Am J Transplant. 2012;12:2774-80.

26. Watson C, Wells A, Roberts R, et al. Cold Machine perfusion versus static cold storage of kidneys donated after cardiac death: a UK multicenter randomized controlled trial. Am J Transplant. 2010;10: 1991-9.

27. Treckmann J, Moers C, Smits J, et al. Machine perfusion versus cold storage for preservation of kidneys from expanded criteria donors after brain death. Transpl Int. 2011;24(6):548-54.

28. O'Callaghan J, Morgan R, Knight S, et al. Systematic review and meta-analysis of hypothermic machine perfusion versus static cold 
storage of kidney allografts on transplant outcomes. Br J Surg. 2013;100:991-1001.

29. Hoyer DP, Gallinat A, Swoboda S, et al. Subnormothermic machine perfusion for preservation of porcine kidneys in a donation after circulatory death model. Transpl Int. 2014;27(10):1097106.
30. Gage F, Leeser DB, Porterfield NK, et al. Room temperature pulsatile perfusion of renal allografts with Lifor compared with hypothermic machine pump solution. Transplant Proc. 2009;41:3571-4.

31. Groen H, Moers C, Smits J, et al. Cost-effectiveness of hypothermic machine preservation versus static cold storage in renal transplantation. Am J Transplant. 2012;12(7):1824-30. 